\title{
EXPLORATORY SIMULATION OF SOLAR GRANULES: HOW SHARP IS THE CONVECTION/RADIATION TRANSITION?
}

\author{
KWING L. CHAN \\ The Hong Kong University of Science and Technology, Hong Kong \\ AND \\ Y. C. KIM \\ Yale University, USA
}

\section{Introduction}

Currently, the most successful direct simulation of the solar granules (and the convection/radiation transition layer) is the three-dimensional (3D) model computed by Stein and Nordlund (1989). So far, there is no other similar $3 \mathrm{D}$ models available for comparison [however, see Ludwig et al. (1997) for a recent $2 \mathrm{D}$ calculation]. We are developing an alternative numerical approach to simulate the $3 \mathrm{D}$ radiation hydrodynamics of this layer. In this approach, the Eddington approximation is used to handle the radiation rather than solving the radiative transfer equations along rays, and the ADISM method (Chan and Wolff 1982) which solves the Navier Stokes equations in conservative forms is used to speed up the thermal relaxation of the fluid layer. We are in the process of testing the numerical accuracy of the codes. This paper summarizes the results of a test that illustrate the effects of vertical space resolution on the mean profiles of some important quantities.

\section{The Model and Results}

The computed domain is a rectangular box $1500 \mathrm{~km}$ wide and $1000 \mathrm{~km}$ deep, with approximately $200 \mathrm{~km}$ above the $\tau$ (optical depth) $\sim 1$ level. We perform calculations with three different meshes: $45 \times 45 \times 83,45 \times 45 \times 42$, and $45 \times 45 \times$ 21. The vertical resolutions for these meshes are $13.3 \mathrm{~km}, 26.6 \mathrm{~km}$, and $53.3 \mathrm{~km}$. The computed mean temperature $(T)$ and flux of kinetic energy $\left(F_{k e}\right)$ profiles are shown in Figs 1 and 2. The solid, dashed, and dotted (with plus signs to 


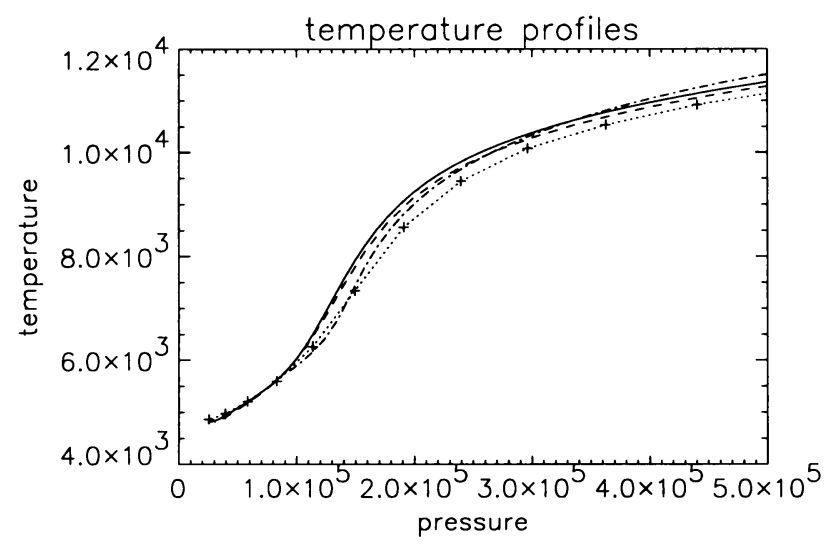

Figure 1. Mean temperature profile for different resolutions

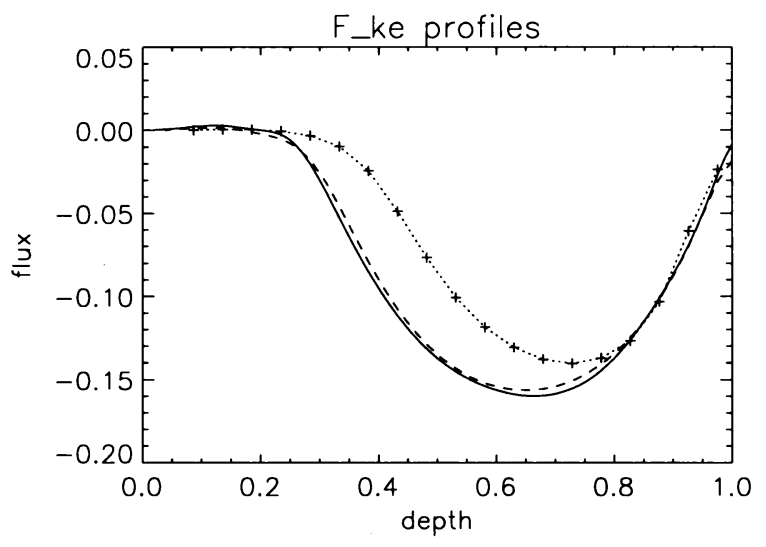

Figure 2. Flux of kinetic energy profiles for different resolutions

show the grid locations) curves are for the high, medium, and low resolution cases, respectively. The dot-dashed curve in Fig 1 shows a profile based on the standard mixing-length theory which is not far off. The high and medium resolution results are close to each other, while the low resolution result differs more significantly, by about $500 \mathrm{~K}$ in the deeper region. In Fig 2 the flux unit is the total energy flux. The low resolution has a relatively more serious effect on $F_{k e}$.

\section{References}

Chan, K. L., and Wolff, C. L. 1982, J. Comput. Phys., 47, 109 Ludwig, H.-G., Freytag, B., and Steffen, M. 1997, this volume Stein, R. F., and Nordlund, A. 1989, Astrophys. J., 342, L95 